https://doi.org/10.22319/rmcp.v12i4.5310

Nota de investigación

\title{
Prevalencia de diversos serovares de Leptospira interrogans en vacas no vacunadas en los estados de Puebla, Tabasco y Veracruz, México
}

Jorge Víctor Rosete Fernández ${ }^{\text {a }}$

Ángel Ríos Utrera ${ }^{\mathrm{b} *}$

Juan P. Zárate Martínez ${ }^{\mathrm{b}}$

Guadalupe A. Socci Escatell ${ }^{\mathrm{c}}$

Abraham Fragoso Islas a

Francisco T. Barradas Piña ${ }^{b}$

Sara Olazarán Jenkins ${ }^{\text {a }}$

Lorenzo Granados Zurita ${ }^{\mathrm{d}}$

${ }^{a}$ Instituto Nacional de Investigaciones Forestales, Agrícolas y Pecuarias (INIFAP). Sitio Experimental Las Margaritas. Kilómetro 9.5 carretera Hueytamalco-Tenampulco, Hueytamalco, Puebla, México.

${ }^{\mathrm{b}}$ INIFAP, Campo Experimental La Posta. Veracruz, México.

${ }^{\mathrm{c}}$ INIFAP, CENID Salud Animal e Inocuidad. Ciudad de México, México.

d INIFAP, Campo Experimental Huimanguillo. Tabasco, México.

*Autor de correspondencia: rios.angel@inifap.gob.mx

\section{Resumen:}

El objetivo fue comparar las prevalencias de anticuerpos contra diferentes serovares de Leptospira interrogans entre los estados de Puebla, Tabasco y Veracruz, así como entre algunos de sus municipios, y determinar si el estatus sanitario de las vacas influye en su 
fertilidad. Se tomaron muestras de sangre de 423 vacas (Bos taurus x Bos indicus y Bos indicus) de 24 ranchos de 11 municipios de los estados mencionados. Las prevalencias de los serovares Hardjo e Inifap fueron mayores $(P<0.05)$ en el estado de Veracruz que en el estado de Puebla, pero la prevalencia del serovar Wolffi fue mayor $(P<0.05)$ en el estado de Puebla que en el estado de Veracruz. Las prevalencias de los serovares Hardjo y Palo Alto fueron mayores $(P<0.05)$ en el estado de Tabasco que en el estado de Puebla, pero no hubo diferencias entre estos dos estados en las prevalencias de los serovares Inifap y Wolffi $(P>0.05)$. El número de serovares en el estado de Veracruz fue mayor $(\mathrm{P}<0.05)$ que en el estado de Puebla, pero el número de serovares en Tabasco fue intermedio; además, existió una variación importante $(P<0.05)$ entre municipios y entre ranchos en la prevalencia de los diferentes serovares. Globalmente, el serovar con mayor frecuencia fue Inifap, mientras que el serovar con menor frecuencia fue Tarassovi. El estatus zoosanitario de las vacas no influyó la tasa de gestación $(P>0.05)$; sin embargo, se recomienda la vacunación del ganado contra Leptospira interrogans, con el fin de disminuir los riesgos asociados con esta bacteria en bovinos y humanos.

Palabras clave: Prevalencia, Leptospira interrogans, Hardjo, Wolffi, Tarassovi, Vacas, Tasa de gestación.

Recibido: 15/04/2019

Aceptado: 30/03/2021

La leptospirosis es una enfermedad infecciosa de origen bacteriano clasificada como una zoonosis distribuida mundialmente, que afecta a numerosas especies de animales domésticos y silvestres ${ }^{(1)}$. Es una enfermedad de gran impacto social y económico en la ganadería, en especial en bovinos, debido a las pérdidas que ocasiona por abortos, mortalidad perinatal, nacimiento de crías débiles, infertilidad y disminución de la producción láctea ${ }^{(2)}$. Existen dos especies: Leptospira interrogans, que es patógena, y Leptospira biflexa, que es saprófita y se localiza en la superficie del suelo y el agua. La Leptospira interrogans es patógena para el hombre y los animales, siendo identificados más de 250 serovares, mientras que la Leptospira biflexa presenta 60 serovares $^{(3)}$. A nivel nacional y mundial, se sabe que la leptospirosis bovina es ocasionada principalmente por el serovar Hardjo, cuyo huésped de mantenimiento es el bovino; sin embargo, en diferentes estudios se ha demostrado que los serovares Hardjo, Wolffi y Tarassovi son los más frecuentes en México ${ }^{(3)}$, aunque se han detectado las leptospiras santarosai y kirschneri con impacto potencial en bovinos ${ }^{(4)}$. El conocimiento de las prevalencias en una localidad, la determinación de sus huéspedes de mantenimiento y el monitoreo de la emergencia de nuevos serovares de Leptospira, son esenciales para comprender la epidemiologia de la leptospirosis en una región y para enfocar las estrategias 
de control ${ }^{(5)}$. Con base en lo anterior, el objetivo fue comparar las prevalencias de anticuerpos contra diferentes serovares de Leptospira interrogans entre los estados de Puebla, Tabasco y Veracruz, así como entre algunos de sus municipios, y determinar si el estatus sanitario de las vacas influye en su fertilidad.

El presente estudio se llevó a cabo en 24 ranchos dedicados a la producción bovina. Seis ranchos se encontraban localizados en tres municipios del estado de Tabasco; once se encontraban en cinco municipios del estado de Puebla; y siete estaban ubicados en tres municipios del estado de Veracruz. Once de los ranchos muestreados producían leche y becerros (sistema doble propósito), mientras que trece ranchos solo producían becerros (sistema vaca-cría).

Los ranchos se seleccionaron con base en un muestreo no probabilístico por conveniencia, de acuerdo con el interés de los ganaderos de participar en el presente estudio. Por otro lado, el tamaño de muestra dependió del presupuesto del estudio, por lo tanto, no todas las vacas de cada rancho se muestrearon; sin embargo, por lo menos 12 vacas se seleccionaron en cada uno. Dentro de cada rancho, las vacas se seleccionaron mediante selección aleatoria simple.

Las hembras utilizadas en el estudio tenían uno o más partos, siendo la mayoría de ellas Bos taurus x Bos indicus, aunque también se utilizaron algunas vacas puras Bos indicus de la raza Brahman ( $\mathrm{N}=403$ y 20, respectivamente). Las hembras no mostraron signos clínicos de ninguna enfermedad en el momento en que se realizaron los muestreos. La presente investigación solo incluyó vacas adultas, ya que no se contó con suficiente recurso económico para muestrear becerros (ambos sexos), toretes y vaquillas. Sin embargo, debido a que las vacas permanecen más tiempo en el hato, tienen mayor probabilidad de infectarse y, en consecuencia, más probabilidad de presentar anticuerpos contra todo tipo de enfermedades.

Las vacas no tenían antecedentes de vacunación contra leptospirosis previo a la realización del estudio, por lo que es válido asumir que la presencia de anticuerpos en las hembras se debió únicamente a la exposición natural a la bacteria. Las vacas se evaluaron reproductivamente por medio de ultrasonografía (vía rectal) de útero y ovarios, con el fin de determinar su estatus reproductivo (gestante o no gestante); sin embargo, en los hatos del estado de Tabasco no fue posible determinar dicho estatus.

Las muestras de sangre se obtuvieron por punción de la vena coccígea. Para obtener el suero de cada una de las muestras de sangre, éstas se centrifugaron a 3,000 xg durante $10 \mathrm{~min}$. Las muestras de suero se conservaron a $-20{ }^{\circ} \mathrm{C}$. El diagnóstico serológico para la detección de anticuerpos contra Leptospira se realizó mediante la técnica de microaglutinación (MAT) ${ }^{(6,7)}$. Para este propósito se incluyeron cinco cepas, tres de referencia internacional (Hardjo, Wolffi y Tarassovi) y dos aislamientos nacionales (Inifap y Palo Alto; serovares Hardjo e Icterohaemorrhagiae, respectivamente) obtenidos en el INIFAP. Esta técnica se realizó en 
microplacas de 96 pozos; se utilizaron $50 \mu 1$ de cada dilución del suero a partir de 1:50 hasta la última dilución doble en donde se observó $50 \%$ de aglutinación en el campo, en solución amortiguadora de fosfatos (PBS). Como antígeno se adicionaron $50 \mu \mathrm{l}$ de cada cepa de Leptospira cultivadas en medio EMJH durante 8 días con un título de $2 \mathrm{X} 10^{8} / \mathrm{ml}$. Tras una hora de incubación a temperatura ambiente, las reacciones fueron observadas en un microscopio de campo oscuro. Todos los sueros que mostraron $50 \%$ o más de aglutinación a una dilución de 1:100 o más fueron considerados positivos. Cuando un suero reaccionó a dos o más antígenos, para el análisis de los datos se tomó el título más alto del suero.

Se analizaron siete variables de respuesta: prevalencia de anticuerpos contra los serovares Hardjo, Inifap, Palo Alto, Wolffi y Tarassovi; número de serovares por vaca; y tasa de gestación. La positividad de anticuerpos se registró como 1 cuando una vaca tuvo anticuerpos contra un serovar de Leptospira interrogans en particular (Hardjo, Inifap, Palo Alto, Wolffi o Tarassovi), mientras que la negatividad de anticuerpos se registró como 0 . Al igual que cada una de las cinco prevalencias de anticuerpos, la tasa de gestación también se registró como una variable binaria. La tasa de gestación se codificó como 1 cuando una vaca resultó gestante al diagnóstico por ultrasonografía rectal; en caso contrario (no gestante), esta variable reproductiva se codificó como 0.

El estudio se realizó bajo un diseño completamente al azar. Con el fin de determinar el efecto de los factores estado de la República Mexicana, municipio anidado en estado de la República Mexicana, y rancho anidado en estado de la República Mexicana x municipio sobre las prevalencias de anticuerpos contra los serovares de Leptospira interrogans se utilizó un modelo de regresión logística. Los análisis se realizaron con el procedimiento GENMOD (PROC GENMOD) del programa $\mathrm{SAS}^{(8)}$, considerando una distribución binomial y aplicando una función liga logit. El número de serovares por vaca se analizó de una manera muy similar a las prevalencias, con la única diferencia de que se consideró una distribución Poisson, ya que esta variable es de tipo conteo. La tasa de gestación también se analizó mediante regresión logística, con el procedimiento GENMOD de $\mathrm{SAS}^{(8)}$, considerando una distribución binomial y aplicando una función liga logit. Para el análisis de esta variable reproductiva, el modelo estadístico incluyó los efectos fijos de estatus sanitario de la vaca, estado de la República Mexicana y municipio anidado en estado de la República Mexicana. El estatus zoosanitario se definió como la presencia/ausencia de anticuerpos contra cualquiera de los cinco serovares de Leptospira interrogans. Cuando una vaca presentó anticuerpos contra al menos uno de los cinco serovares, el estatus sanitario se registró como seropositivo; cuando una vaca no presentó anticuerpos contra ninguno de los serovares de Leptospira interrogans, el estatus zoosanitario se registró como seronegativo. El criterio de convergencia fue $10^{-8}$ en los siete análisis estadísticos.

La prevalencia del serovar Hardjo en los estados de Tabasco y Veracruz fue mayor $(P<0.05)$ que en el estado de Puebla (64.1 y $71.3 \%$ vs $39.8 \%$; Figura 1). La prevalencia del serovar 
Inifap fue mayor $(P<0.05)$ en el estado de Veracruz que en los estados de Puebla y Tabasco, con valores de $91.8,63.8$ y $78.8 \%$, respectivamente. Estos valores son considerablemente mayores que los reportados en la literatura científica para el serovar Hardjo identificado en bovinos de los estados de Campeche ${ }^{(9)}$, Oaxaca ${ }^{(10,11)}$, Yucatán ${ }^{(12-14)}$, Veracruz ${ }^{(15,16)}$, Tamaulipas ${ }^{(17)}$ y Estado de México ${ }^{(18)}$.

Figura 1: Prevalencias de serovares de Leptospira interrogans, por estado

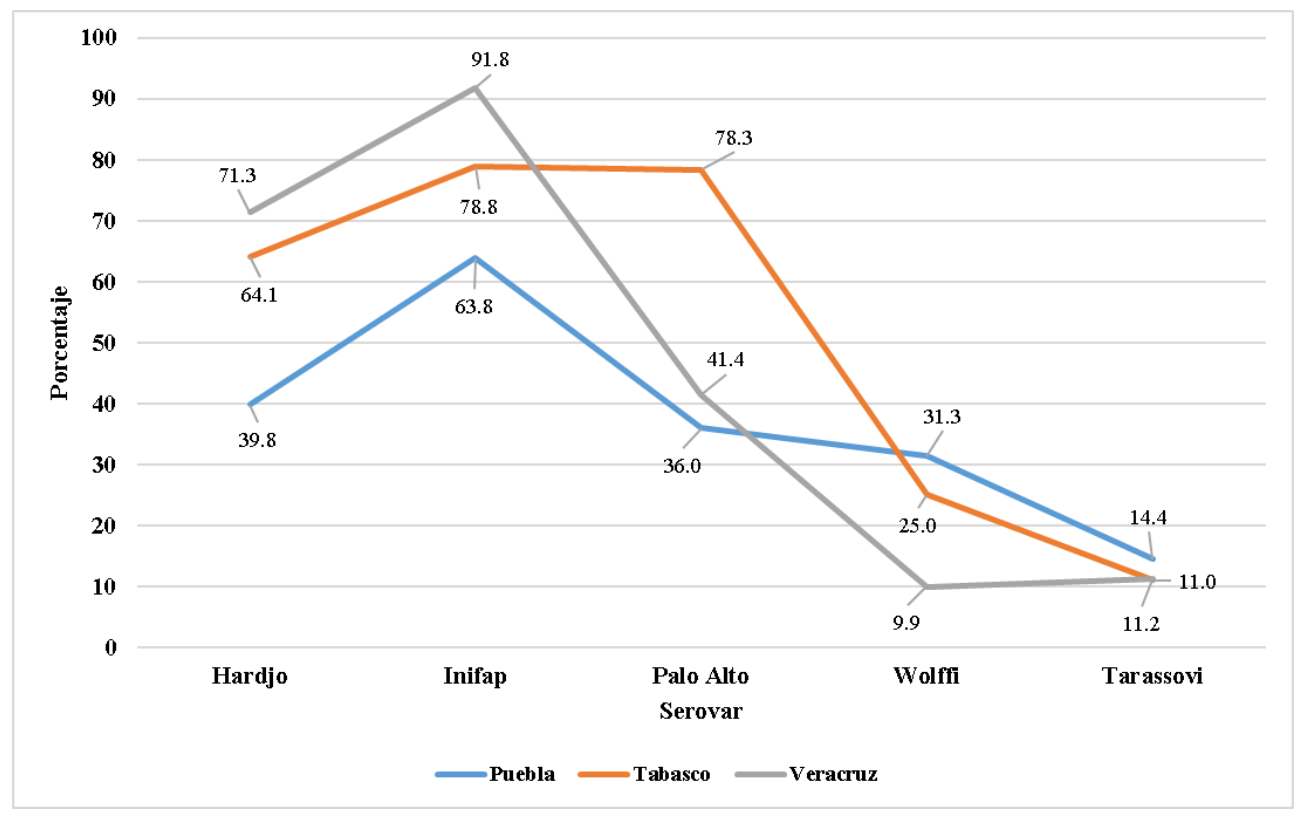

La prevalencia del serovar Palo Alto fue mayor $(P<0.05)$ en el estado de Tabasco que en los estados de Puebla y Veracruz (78.3 vs 36.0 y $41.4 \%$ ). La prevalencia del serovar Palo Alto para el estado de Veracruz reportada en el presente estudio es dos veces mayor que la encontrada $(19.8 \%)$ en un estudio previo realizado en el sur de este mismo estado ${ }^{(16)}$. La prevalencia promedio del serovar Palo Alto $(51.9 \%)$ obtenida en el presente estudio es similar a la reportada por Ramos $e a^{(10)}$ para el estado de Oaxaca (57.1\%), pero mucho mayor que las reportadas $(8.8,8.0,3.1,1.6,1.0$ y $0.0 \%)$ por otros autores para otros estados de la República Mexicana ${ }^{(13,14,19,20,21)}$.

La prevalencia del serovar Wolffi en el estado de Puebla fue mayor $(P<0.05)$ que en el estado de Veracruz (31.3 vs $9.9 \%$ ); la prevalencia del serovar Wolffi en el estado de Tabasco (25.0 \%) fue similar a las prevalencias encontradas en los estados de Puebla y Veracruz. La prevalencia del serovar Wolffi obtenida en el presente trabajo para el estado de Veracruz es similar a la prevalencia promedio correspondiente $(11.0 \%)$ encontrada en un estudio anterior realizado en cuatro municipios del sur de este estado ${ }^{(16)}$. En diversos estudios realizados en otros estados de la República Mexicana (Yucatán, Oaxaca, Estado de México) también se han hallado bajas prevalencias (menores a $10 \%$ ) para el serovar Wolffi ${ }^{(11,12,14,18)}$. Por el 
contrario, las prevalencias del serovar Wolffi $(77.7$ y $66.0 \%)$ reportadas por Córdova et a ${ }^{(9)}$ y Luna et $\mathrm{l}^{(19)}$ son, al menos, dos veces más grandes que las prevalencias del serovar Wolffi reportadas en la presente investigación para los estados de Puebla y Tabasco.

Las prevalencias del serovar Tarassovi encontradas en los estados de Puebla, Tabasco y Veracruz fueron similares entre sí $(P<0.01)$, con valores de $14.4,11.0$ y $11.2 \%$, respectivamente. Estas prevalencias son similares a las reportadas previamente para los estados de Veracruz ${ }^{(16)}$ y Tamaulipas ${ }^{(17)}$, pero mucho menores que las reportadas por Cárdenas-Marrufo et $a l^{(12)}$, Segura-Correa $e t$ al ${ }^{(13)}$ y Luna et $a l^{(19)}$, quienes reportaron prevalencias para el serovar Tarassovi con valores de 66.6, 53.6 y $53.3 \%$, respectivamente. Por el contrario, otros autores han reportado prevalencias para este mismo serovar menores a $8.0 \% \%^{(9,10,11,18,21)}$.

Las prevalencias promedio de los serovares Hardjo, Inifap, Palo Alto, Wolffi y Tarassovi fueron 58.4, 78.1, 51.9, 22.1 y $12.2 \%$, respectivamente, lo que indica que el serovar Inifap fue el más frecuente, mientras que los serovares Wolffi y Tarassovi fueron los menos frecuentes. Este orden de magnitud de las prevalencias es similar al encontrado en Oaxaca ${ }^{(11)}$ y Campeche ${ }^{(9)}$. Por el contrario, en Tamaulipas, Veracruz y Estado de México se encontró que los serovares Wolffi, Tarassovi y Hardjo tuvieron similares prevalencias ${ }^{(16-18)}$. En el estado de Yucatán se determinó una mayor prevalencia para el serovar Tarassovi (53.6\%) que para los serovares Hardjo (31.6\%) y Wolffi $(9.4 \%)^{(12)}$, resultado que también difiere del obtenido en el presente trabajo.

El número de serovares de Leptospira interrogans presentes en las vacas del estado de Veracruz fue mayor $(P<0.05)$ que el número de serovares presentes en las vacas del estado de Puebla (2.23 vs 1.68); el número de serovares de Leptospira interrogans presentes en las vacas del estado de Tabasco fue intermedio (2.05). De manera general, se esperaba encontrar una mayor prevalencia de cada uno de los serovares estudiados y un mayor número de serovares por vaca en los estados de Veracruz y Tabasco que en el estado de Puebla, ya que en los estados de Veracruz y Tabasco existe mayor precipitación pluvial y temperatura ambiental que en el estado de Puebla; sin embargo, el serovar Hardjo fue el único serovar que presentó mayor prevalencia en Veracruz y Tabasco en relación con Puebla (Figura 1). En un estudio que comparó la prevalencia de leptospirosis de las distintas regiones ecológicas de México, se encontró que la prevalencia fue mayor en las regiones tropical seca y tropical húmeda que en las regiones árida/semiárida y templada ${ }^{(19)}$; sin embargo, este estudio careció de un análisis estadístico formal. Vinetz ${ }^{(22)}$ informó que la Leptospirosis ocurre con mayor frecuencia en países con clima tropical, donde la precipitación pluvial y la temperatura ambiental son mayores.

En el estado de Puebla, los municipios de Ayotoxco, Hueytamalco y San José Acateno presentaron prevalencias del serovar Hardjo mayores $(P<0.05)$ que el municipio de 
Nauzontla. En el estado de Tabasco, las prevalencias del serovar Hardjo de los municipios de Cunduacán, Huimanguillo y Ranchería El Puente fueron similares ( $P>0.05$; Cuadro 1). En el estado de Veracruz, los municipios de Cotaxtla, Medellín y San Rafael presentaron similares prevalencias del serovar Hardjo.

Cuadro 1: Medias de cuadrados mínimos y errores estándar para prevalencia (\%) y número de serovares de Leptospira interrogans, por municipio

\begin{tabular}{|c|c|c|c|c|c|c|c|c|c|c|c|}
\hline \multirow{4}{*}{$\begin{array}{l}\text { Municipio } \\
\text { Ayotoxco }\end{array}$} & \multicolumn{9}{|c|}{ Serovar } & & \\
\hline & \multicolumn{2}{|c|}{ Hardjo } & \multicolumn{2}{|l|}{ Inifap } & \multicolumn{2}{|c|}{$\begin{array}{l}\text { Palo } \\
\text { Alto }\end{array}$} & \multicolumn{2}{|l|}{ Wolffi } & Tarassovi & \multicolumn{2}{|c|}{ Número } \\
\hline & 65.0 & \pm & 90.0 & \pm & 60.0 & \pm & $80.0 \pm 8$. & & 35.0 & 3.30 & \pm \\
\hline & $10.7^{\mathrm{ab}}$ & & $6.7^{\mathrm{ab}}$ & & $11.0^{\mathrm{ab}}$ & & & & $10.7^{\mathrm{a}}$ & $0.41^{\mathrm{a}}$ & \\
\hline \multirow[t]{2}{*}{ Hueytamalco } & 49.7 & \pm & 68.9 & \pm & 21.4 & \pm & $6.9 \pm 4.1^{c}$ & & $\mathrm{NE}$ & 1.36 & \pm \\
\hline & $5.0^{\mathrm{ab}}$ & & $4.7^{\mathrm{b}}$ & & $4.6^{\mathrm{c}}$ & & & & & $0.12^{\mathrm{e}}$ & \\
\hline \multirow[t]{2}{*}{ Nauzontla } & \multirow{2}{*}{\multicolumn{2}{|c|}{$7.7 \pm 7.4^{\mathrm{c}}$}} & 15.4 & \pm & 46.2 & \pm & 38.5 & \pm & $\mathrm{NE}$ & 1.08 & \pm \\
\hline & & & $10.0^{\mathrm{c}}$ & & $13.8^{\mathrm{b}}$ & & $13.5^{\mathrm{b}}$ & & & $0.29^{\mathrm{e}}$ & \\
\hline San José & 67.5 & \pm & 77.9 & \pm & 17.8 & \pm & 20.9 & \pm & $5.0 \pm 3.4^{\mathrm{c}}$ & 1.77 & \pm \\
\hline Acateno & $7.3^{\mathrm{ab}}$ & & $5.9^{\mathrm{b}}$ & & $5.6^{c}$ & & $5.7^{\mathrm{bc}}$ & & & $0.18^{d}$ & \\
\hline \multirow[t]{2}{*}{ Xochitlán } & 28.6 & \pm & 57.1 & \pm & 42.9 & \pm & 28.6 & \pm & $\mathrm{NE}$ & 1.57 & \pm \\
\hline & $17.1^{\mathrm{bc}}$ & & $18.7^{\mathrm{bc}}$ & & $18.7^{\mathrm{b}}$ & & $17.1^{\mathrm{bc}}$ & & & $0.47^{\mathrm{d}}$ & \\
\hline \multirow[t]{2}{*}{ Cunduacán } & 41.7 & \pm & 58.3 & \pm & 41.7 & \pm & $\mathrm{NE}$ & & $\mathrm{NE}$ & 1.42 & \pm \\
\hline & $14.2^{\mathrm{abc}}$ & & $14.2^{\mathrm{b}}$ & & $14.2^{\mathrm{b}}$ & & & & & $0.34^{\mathrm{de}}$ & \\
\hline \multirow[t]{2}{*}{ Huimanguillo } & 63.8 & \pm & 78.4 & \pm & 86.8 & \pm & 25.0 & \pm & 13.2 & 2.24 & \pm \\
\hline & $6.8^{\mathrm{ab}}$ & & $7.6^{\mathrm{b}}$ & & $5.1^{\mathrm{a}}$ & & $10.8^{\mathrm{bc}}$ & & $5.5^{\mathrm{abc}}$ & $0.22^{\mathrm{bcd}}$ & \\
\hline \multirow[t]{2}{*}{ El Puente } & 81.8 & \pm & 90.9 & \pm & 90.9 & \pm & $\mathrm{NE}$ & & $9.1 \pm 8.7^{\mathrm{bc}}$ & 2.73 & \pm \\
\hline & $11.6^{\mathrm{a}}$ & & $8.7^{\mathrm{ab}}$ & & $8.7^{\mathrm{a}}$ & & & & & $0.50^{\mathrm{a}}$ & \\
\hline \multirow[t]{2}{*}{ Cotaxtla } & 67.1 & \pm & 89.4 & \pm & 70.1 & \pm & $\mathrm{NE}$ & & 27.7 & 2.56 & \pm \\
\hline & $9.9^{\mathrm{ab}}$ & & $5.5^{\mathrm{ab}}$ & & $7.6^{\mathrm{a}}$ & & & & $11.5^{\mathrm{ab}}$ & $0.26^{\mathrm{ab}}$ & \\
\hline \multirow[t]{2}{*}{ Medellín } & 81.7 & \pm & 95.4 & \pm & 40.6 & \pm & $6.5 \pm 3.8^{c}$ & & $9.1 \pm 4.4^{\mathrm{bc}}$ & 2.34 & \pm \\
\hline & $5.8^{\mathrm{a}}$ & & $3.2^{\mathrm{a}}$ & & $8.0^{\mathrm{b}}$ & & & & & $0.23^{b c}$ & \\
\hline \multirow[t]{2}{*}{ San Rafael } & 62.6 & \pm & 88.3 & \pm & 18.0 & \pm & 14.8 & \pm & $5.0 \pm 3.4^{\mathrm{c}}$ & 1.86 & \pm \\
\hline & $6.6^{\mathrm{ab}}$ & & $4.6^{\mathrm{ab}}$ & & $5.3^{\mathrm{c}}$ & & $4.9^{\mathrm{bc}}$ & & & $0.18^{\mathrm{cd}}$ & \\
\hline
\end{tabular}

En el estado de Puebla, los municipios de Ayotoxco, Hueytamalco y San José Acateno presentaron prevalencias del serovar Inifap mayores $(P<0.05)$ que el municipio de Nauzontla. Cunduacán, Huimanguillo y Ranchería El Puente, municipios del estado de Tabasco, tuvieron prevalencias del serovar Inifap similares $(P>0.05)$. Similarmente, los municipios de Cotaxtla, Medellín y San Rafael no presentaron diferencia $(P>0.05)$ en la prevalencia del serovar Inifap (Cuadro 1). 
Los municipios de Ayotoxco, Nauzontla y Xochitlán, del estado de Puebla, presentaron mayores $(P<0.05)$ prevalencias del serovar Palo Alto que los municipios de Hueytamalco y San José Acateno. En Tabasco, las prevalencias del serovar Palo Alto de los municipios de Huimanguillo y Ranchería El Puente fueron mayores $(P<0.05)$ que la del municipio de Cunduacán. En el estado de Veracruz, el municipio de Cotaxtla mostró una mayor $(\mathrm{P}<0.05)$ prevalencia del serovar Palo Alto que los municipios de Medellín y San Rafael (Cuadro 1). Se encontró una mayor $(P<0.05)$ prevalencia del serovar Wolffi en el municipio de Ayotoxco que en los demás municipios del estado de Puebla. Las prevalencias del serovar Wolffi encontradas en los tres municipios del estado de Veracruz fueron similares $(P>0.05$; Cuadro 1).

En el estado de Puebla, el municipio de Ayotoxco tuvo una prevalencia del serovar Tarassovi similar $(P>0.05)$ a la del de San José Acateno. Las prevalencias del serovar Tarassovi encontradas en el estado de Tabasco no fueron diferentes entre sí $(P>0.05)$. En el estado de Veracruz, la prevalencia del serovar Tarassovi del municipio de Cotaxtla fue mayor $(P<0.05)$ que la del municipio de San Rafael (Cuadro 1). Al parecer, esta es la primera vez que se reporta la prevalencia de estos cinco serovares para estos once municipios de los estados de Puebla, Tabasco y Veracruz, pues no se encontraron en la literatura publicaciones científicas al respecto.

El número de serovares de Leptospira interrogans encontradas en el municipo de Ayotoxco fue mayor $(P>0.05)$ que el número de serovares encontrados en los demás municipios del estado de Puebla. Se encontró un mayor $(P<0.05)$ número de serovares en el municipio de Ranchería El Puente que en los municipios de Cunduacán y Huimanguillo. El número de serovares encontrados en el municipio de Cotaxtla fue mayor $(P<0.05)$ que el número de serovares encontrados en el de San Rafael (Cuadro 1).

Las vacas con anticuerpos contra Leptospira interrogans tuvieron una tasa de gestación similar $(P>0.05)$ a las vacas sin anticuerpos (55.5 vs $51.5 \%)$; tampoco existió diferencia $(P>0.05)$ en la tasa de gestación entre estados, ni entre municipios (Cuadro 2). En concordancia con este resultado, se ha reportado que Leptospira interrogans no se encuentra relacionada con la generación de quistes foliculares, los cuales afectan negativamente la fertilidad de las vacas ${ }^{(23)}$. 
Cuadro 2: Tasas de gestación (\%) y sus respectivos errores estándar e intervalos de confianza al 95\% (IC95\%) por estatus zoosanitario de la vaca, estado de la República

Mexicana y municipio

\begin{tabular}{|c|c|c|}
\hline Efecto/nivel & Tasa de gestación & IC95\% \\
\hline \multicolumn{3}{|l|}{ Estatus zoosanitario } \\
\hline Seronegativo ${ }^{a}$ & $51.5 \pm 8.5^{\mathrm{c}}$ & $35.2-67.4$ \\
\hline Seropositivo ${ }^{\mathrm{b}}$ & $55.5 \pm 3.7^{\mathrm{c}}$ & $48.1-62.6$ \\
\hline \multicolumn{3}{|c|}{ Estado de la República Mexicana } \\
\hline Puebla & $51.4 \pm 6.4^{\mathrm{c}}$ & $38.9-63.7$ \\
\hline Veracruz & $55.5 \pm 5.8^{\mathrm{c}}$ & $44.0-66.5$ \\
\hline \multicolumn{3}{|l|}{ Municipio } \\
\hline Ayotoxco de Guerrero & $48.2 \pm 11.8^{\mathrm{c}}$ & $26.9-70.2$ \\
\hline Hueytamalco & $51.2 \pm 6.0^{c}$ & $39.7-62.7$ \\
\hline Nauzontla & $38.0 \pm 13.5^{\mathrm{c}}$ & $16.7-65.3$ \\
\hline San José Acateno & $48.8 \pm 7.0^{c}$ & $35.6-62.2$ \\
\hline Xochitlán & $69.8 \pm 18.0^{c}$ & $30.2-92.5$ \\
\hline Cotaxtla & $49.4 \pm 9.0^{c}$ & $32.5-66.4$ \\
\hline Medellín de Bravo & $68.0 \pm 8.1^{\mathrm{c}}$ & $50.6-81.6$ \\
\hline San Rafael & $48.4 \pm 7.3^{\mathrm{c}}$ & $34.6-62.5$ \\
\hline
\end{tabular}

En conclusión, las prevalencias de los serovares Hardjo e Inifap fueron mayores en el estado de Veracruz que en el estado de Puebla, pero la prevalencia del serovar Wolffi fue mayor en el estado de Puebla que en el estado de Veracruz. Las prevalencias de los serovares Hardjo y Palo Alto fueron mayores en el estado de Tabasco que en el estado de Puebla, pero no hubo diferencias entre estos dos estados en las prevalencias de los serovares Inifap y Wolffi. El número de serovares de Leptospira interrogans en el estado de Veracruz fue mayor que en el estado de Puebla, pero el número de serovares en el estado de Tabasco fue intermedio; además, entre municipios y entre ranchos existió una variación importante en la prevalencia de los diferentes serovares de Leptospira interrogans. De manera global, el serovar con la mayor frecuencia fue Inifap, mientras que el serovar con la menor frecuencia fue Tarassovi. El estatus sanitario de las vacas no influyó la fertilidad de las mismas; sin embargo, los ganaderos de los municipios evaluados deberían vacunar contra Leptospira interrogans, como medida preventiva para disminuir los riesgos asociados con esta bacteria en bovinos y humanos. 


\section{Literatura citada:}

1. Dragui MG, Brihuega B, Benítez D, Sala JM, Biotti GM, Pereyra M, Homse A, Guariniello L. Brote de leptospirosis en terneros en recría en la provincia de Corrientes, Argentina. Rev Arg Microbiol 2011;43:42-44.

2. Arias ChF, Suárez AF, Huanca LW, Rivera GH, Camacho SJ, Huanca MT. Prevalencia de leptospirosis bovina en dos localidades de Puno en época de seca y determinación de factores de riesgo. Rev Inv Vet Perú 2011;22(2):167-170.

3. Méndez C, Benavides L, Esquivel A, Aldama A, Torres J, Gavaldón D, Meléndez P, Moles L. Pesquisa serológica de Leptospira en roedores silvestres, bovinos, equinos y caninos en el noreste de México. Rev Salud Anim 2013;35(1):25-32.

4. Carmona-Gasca CA, León LL, Castillo-Sánchez LO, Ramírez-Ortega JM, Ko A, Luna PC, de la Peña-Moctezuma A. Detección de Leptospira santarosai y L. kirschneri en bovinos: nuevos aislados con potencial impacto en producción bovina y salud pública. Vet Méx 2011;42(4):277-288.

5. Hernández-Rodríguez P, Gómez AP, Villamil LC. Implicaciones de las prácticas agropecuarias urbanas y rurales sobre la transmisión de la leptospirosis. Agrociencia 2017;51:725-741.

6. OIE. Organización Mundial de Sanidad Animal. 2004. Manual de las pruebas de diagnóstico y de las vacunas para los animales terrestres (mamíferos, aves y abejas). http://www.oie.int/doc/ged/D6508.pdf. Consultado 8 abr, 2019.

7. WHO. World Health Organization. 2019. Zoonoses. Leptospirosis. http://www.who.int/zoonoses/diseases/leptospirosis/en/. Consultado 8 abr, 2019.

8. SAS Institute Inc. SAS/STAT® 9.3 User's guide. Cary, NC: SAS Institute Inc. 2011.

9. Córdova IA, Cano MS, Moles CLP, Cisneros PMA, Rodríguez AG, Ávila GJ, Pérez GJF. Diagnóstico de leptospirosis en ganado bovino productor de carne. REDVET 2005;6(7):1-5.

10. Ramos GAB, Herrera LE, Gutiérrez HJL, Palomares REG, Díaz AE, Limón GMM, et al. Frecuencia de rinotraqueitis infecciosa bovina (IBR), diarrea viral bovina (DVB), y leptospirosis, en bovinos de doble propósito, en el municipio de San Juan Cotzocón, Oaxaca, México. En: Ricardo GID, et al, editores. Congreso Nacional de Buiatría. Villahermosa, Tabasco, México. 2014:134-139. 
11. Hernández BEG, Gutiérrez HJL, Herrera LE, Palomares REG, Díaz AE. Frecuencia de diarrea viral bovina, rinotraqueitis infecciosa bovina, leptospirosis y brucelosis, en las dos regiones ganaderas más importantes de Oaxaca. En: Ricardo GID, Posadas ME editores. Congreso Nacional de Buiatría. Puebla, Puebla, México. 2015:87-92.

12. Cárdenas-Marrufo MF, Vado-Solís I, Pérez-Osorio CE, Segura-Correa JC. Seropositivity to leptospirosis in domestic reservoirs and detection of Leptospira spp. from water sources, in farms of Yucatan, Mexico. Trop Subtrop Agroecosys 2011;14:185-189.

13. Segura-Correa VM, Solis-Calderón JJ, Segura-Correa JC. Seroprevalence of and risk factors for leptospiral antibodies among cattle in the state of Yucatan, Mexico. Trop Anim Hlth Prod 2003;35:293-299.

14. Vado-Solís I, Cárdenas-Marrufo MF, Jiménez-Delgadillo B, Alzina-López A, LaviadaMolina H, Suarez-Solís V, Zavala-Velázquez JE. Clinical-epidemiological study of Leptospirosis in humans and reservoirs in Yucatán, México. Rev Inst Med Trop Sao Paulo 2002;44(6):335-340.

15. Barajas-Rojas JA, Riemann HP, Franti CE. Application of enzyme-linked immunosorbent assay for epidemiological studies of diseases of livestock in the tropics of Mexico. Rev Sci Tech Off Int Epiz 1993;12(3):717-732.

16. Rodríguez BSA. Serofrecuencia de leptospirosis bovina en cuatro municipios ubicados en el sur del estado de Veracruz [tesis maestría]. Veracruz, Ver.: Universidad Veracruzana; 2010.

17. Cantú CA, Banda RVM. Serofrecuencia de leptospirosis bovina en tres municipios del sur de Tamaulipas. Tec Pecu Mex 1995;33(2):121-124.

18. Leon LL, Garcia RC, Diaz CO, Valdez RB, Carmona GCA, Velazquez BLG. Prevalence of Leptospirosis in dairy cattle from small rural production units in Toluca Valley, State of Mexico. Animal Biodiversity and Emerging Diseases: Ann NY Acad Sci 2008;1149:292-295.

19. Luna AMA, Moles CLP, Gavaldón RD, Nava VC, Salazar GF. Estudio retrospectivo de serofrecuencia de leptospirosis bovina en México considerando las regiones ecológicas. Rev Cubana Med Trop 2005;57(1):28-31.

20. Zavala VJ, Pinzón CJ, Flores CM, Damián CAG. La Leptospirosis en Yucatán. Estudio serológico en humanos y animales. Salud Púb Méx 1984;26:254-59.

21. Moles CLP, Cisneros PMA, Gavaldón RD, Rojas SN, Torres BJI. Estudio serológico de leptospirosis bovina en México. Rev Cubana Med Trop 2002;54(1):24-27. 
22. Vinetz JM. Leptospirosis. Current opinion in infectious diseases. 2001;14(5):527-538.

23. Cedillo SLC, Banda RVM, Morales SE, Villagómez-Amezcua ME. Asociación de quistes foliculares ováricos con la presencia de anticuerpos y agentes causantes de las principales enfermedades infecciosas reproductivas en vacas. Abanico Vet 2012;2(1):11-22. 